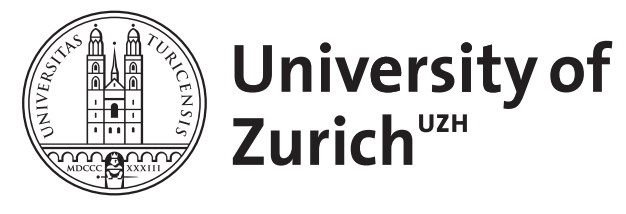

\title{
LHC Lead Data and Nuclear PDFs
}

Kusina, A ; Lyonnet, F ; Clark, D B ; Godat, E ; Ježo, T ; Kovař́́k, K ; Olness, F I ; Schienbein, I ; Yu, J Y

\begin{abstract}
We compare predictions of nCTEQ15 nuclear parton distribution functions with proton-lead vector boson production data from the LHC. We select data sets that are most sensitive to nuclear PDFs and have potential to constrain them. We identify the kinematic regions and flavors where these data can bring new information and will have largest impact on the nuclear PDFs. Finally, we estimate the effect of including these data in a global analysis using a reweighting method.
\end{abstract}

DOI: https://doi.org/10.5506/APhysPolB.48.1035

Posted at the Zurich Open Repository and Archive, University of Zurich ZORA URL: https://doi.org/10.5167/uzh-149630

Journal Article

Published Version

Originally published at:

Kusina, A; Lyonnet, F; Clark, D B; Godat, E; Ježo, T; Kovař́k, K; Olness, F I; Schienbein, I; Yu, J Y (2017). LHC Lead Data and Nuclear PDFs. Acta Physica Polonica B, 48(6):1035-1047.

DOI: https://doi.org/10.5506/APhysPolB.48.1035 


\title{
LHC LEAD DATA AND NUCLEAR PDFs*
}

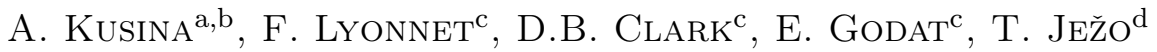 \\ K. Kovarí́K ${ }^{\mathrm{e}}$, F.I. Olness ${ }^{\mathrm{c}}$, I. Schienbein ${ }^{\mathrm{a}}$, J.Y. Yu ${ }^{\mathrm{c}}$ \\ ${ }^{a}$ Laboratoire de Physique Subatomique et de Cosmologie \\ 53 Rue des Martyrs Grenoble, France \\ ${ }^{\mathrm{b}}$ The H. Niewodniczański Institute of Nuclear Physics, Polish Academy of Sciences \\ Radzikowskiego 152, 31-342 Kraków, Poland \\ ${ }^{\mathrm{c}}$ Southern Methodist University, Dallas, TX 75275, USA \\ ${ }^{d}$ Physik-Institut, Universität Zürich \\ Winterthurerstrasse 190, 8057 Zürich, Switzerland \\ ${ }^{\mathrm{e}}$ Institut für Theoretische Physik, Westfälische Wilhelms-Universität Münster \\ Wilhelm-Klemm-Straße 9, 48149 Münster, Germany
}

\section{(Received April 21, 2017)}

We compare predictions of nCTEQ15 nuclear parton distribution functions with proton-lead vector boson production data from the LHC. We select data sets that are most sensitive to nuclear PDFs and have potential to constrain them. We identify the kinematic regions and flavors where these data can bring new information and will have largest impact on the nuclear PDFs. Finally, we estimate the effect of including these data in a global analysis using a reweighting method.

DOI:10.5506/APhysPolB.48.1035

\section{Introduction}

Nuclear parton distribution functions (nPDFs) are important quantities necessary to describe high-energy collisions including heavy ions, as well as giving insight into the structure of nuclei. nPDFs are non-perturbative objects that cannot be calculated by the known methods. Instead, similarly to what is done in the case of proton PDFs, nPDFs are extracted from experimental data in the process of global analysis. However, in the nPDF case not only the $x$-dependence is modeled, but also the $A$-dependence (where $A$ is the nucleus mass). This is partly by design to have a general parametrization of different nuclei, and partly by necessity as there is typically not a sufficient amount of experimental data to constrain distributions for individual nuclei separately. The lack of kinematical data is actually one of the

* Presented by A. Kusina at the Cracow Epiphany Conference "Particle Theory Meets the First Data from LHC Run 2", Kraków, Poland, January 9-12, 2017. 
main practical differences between the proton and nuclear PDF fits. This is illustrated in Fig. 1 where we compare the kinematical range of data in both cases. The lack of data is also the reason why in many cases additional assumptions need to be introduced in the nPDF analyses in order to obtain stable fits. This necessity, however, can lead to sizable differences (much bigger than for proton PDFs) between different nPDFs, see Fig. 2.
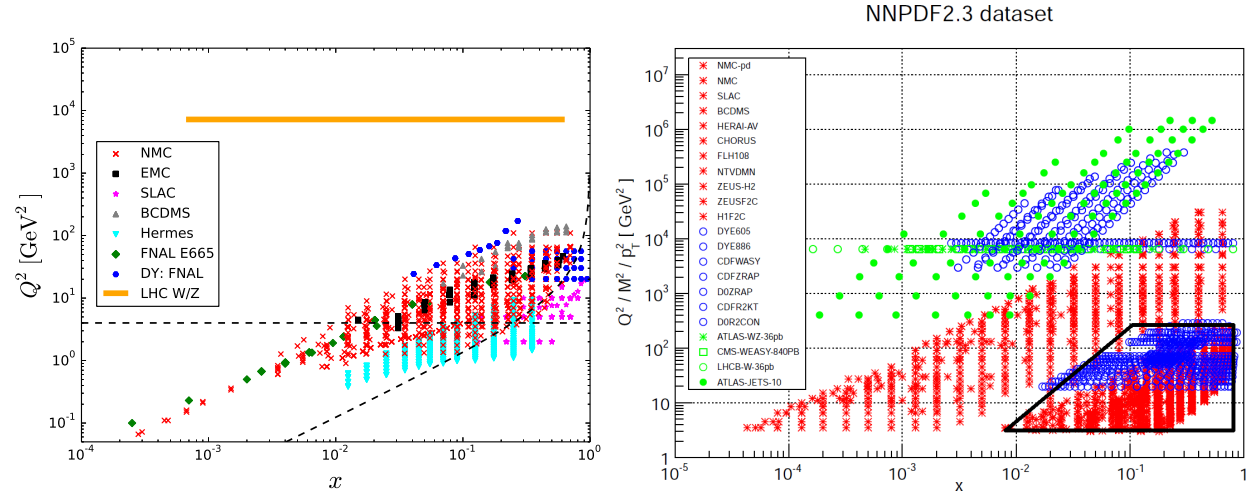

Fig. 1. Typical kinematical range of data used in nPDF global analysis with addition of $W / Z p \mathrm{~Pb}$ LHC data (left). Example kinematical range of data used in free-proton PDF global analysis [1] (right).

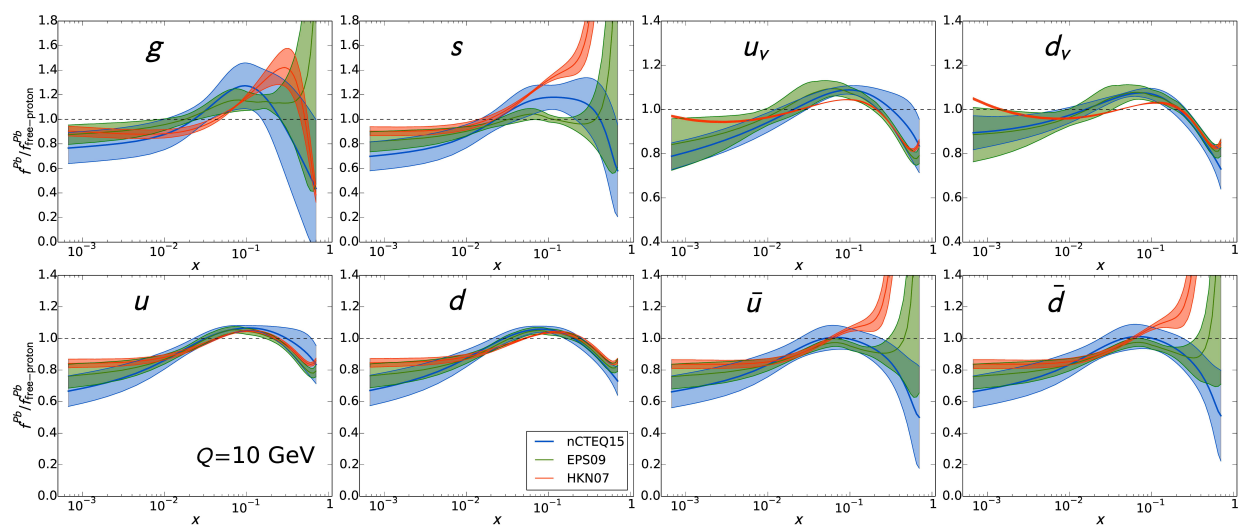

Fig. 2. Comparison of lead nuclear modifications, $R_{i}^{\mathrm{Pb}}=\frac{f^{\mathrm{Pb}}}{f_{\text {free }}^{\mathrm{Pb}} \text { proton }}$, obtained by nCTEQ15 [2], EPS09 [3] and HKN07 [4] nPDF global analyses. Figure from [2].

Vector boson production in hadron collisions is a very well understood process and serves as one of the "standard candle" measurements at the LHC. $W^{ \pm}$and $Z$ bosons are numerously produced in heavy-ion protonlead $(p \mathrm{~Pb})$ and lead-lead $(\mathrm{PbPb})$ collisions at the LHC and can be used to 
gain insight into the structure of nPDFs. As the $W$ and $Z$ bosons couple weakly, their interaction with the nuclear medium is negligible which makes these processes one of the cleanest probes of the nuclear structure available at the LHC. The possibility of using vector boson production data to constrain nPDFs was considered before the LHC data were available [5], and this demonstrated a strong potential for the proton-lead data to constrain nPDFs. The current LHC measurements for $W^{ \pm}$and $Z$ production include mostly rapidity distributions for both $p \mathrm{~Pb}$ and $\mathrm{PbPb}$ collisions [6-15]. Some of these data were already used in a reweighting analyses [16] and, more recently [17], to estimate the impact of these data on EPS09 [3], DSSZ [18] and nCTEQ15 [2] nPDFs. Analysis of these data was also performed within the framework of KP model [19]. Lately, a first global analysis of nPDFs with LHC data, EPPS16 [20], has been published ${ }^{1}$.

In this work, we present predictions for vector boson production in $p \mathrm{~Pb}$ and $\mathrm{PbPb}$ collisions at the LHC obtained using nCTEQ15 nuclear parton distributions, and perform a comprehensive comparison to the available LHC data. We also identify the measurements which have the biggest potential to constrain the nPDFs with a special attention to the strange distribution. Finally, we perform a reweighting study that gives indications on the effects of including these data in the nCTEQ global fit. This proceedings is based on the recent study [17] with additional material on the nuclear strange distribution.

\section{Comparison to the LHC vector boson data}

We start by comparing predictions for vector boson production at the LHC calculated using nCTEQ15 nPDFs [2] to the available experimental data (for the proton beam, we use CT10 proton PDFs [22]). In this note, we concentrate only on the most relevant data sets, namely $W^{ \pm}$production in $p \mathrm{~Pb}$ collisions $(\sqrt{s}=5.02 \mathrm{TeV})$ from CMS [9] and ATLAS [10]. A more comprehensive comparison with all the currently available vector boson data can be found in Ref. [17]. Our calculations are done using next-to-leading order (NLO) with help of the FEWZ [23] program.

In Fig. 3, we present results for CMS [9] and ATLAS [10] data. In the plots, we show data overlaid with predictions using nCTEQ15 nPDFs (darker/blue band) and, additionally, we show results obtained with free proton PDFs (lighter/yellow band) for which we choose CT10 distributions $[22]^{2}$.

${ }^{1}$ Interesting scaling properties of the LHC $W$ production data from $p p, p \mathrm{~Pb}$ and $\mathrm{PbPb}$ collisions have been observed in [21].

${ }^{2}$ We include here the isospin effects. 


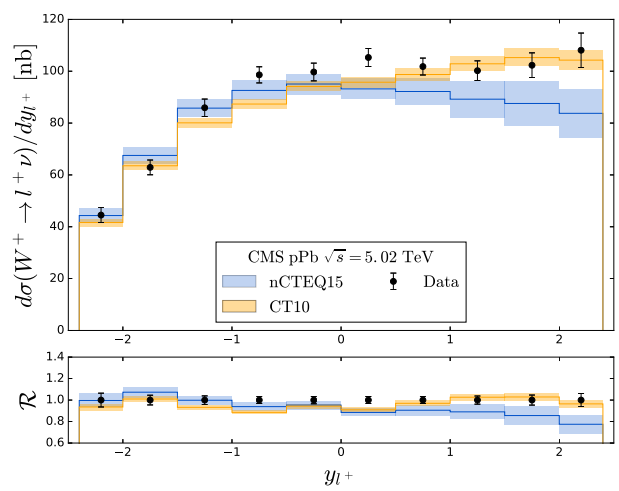

(a) $\mathrm{CMS} W^{+}$

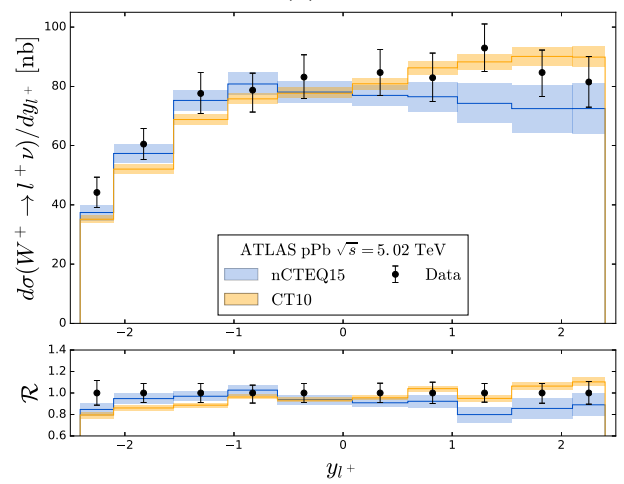

(c) ATLAS $W^{+}$

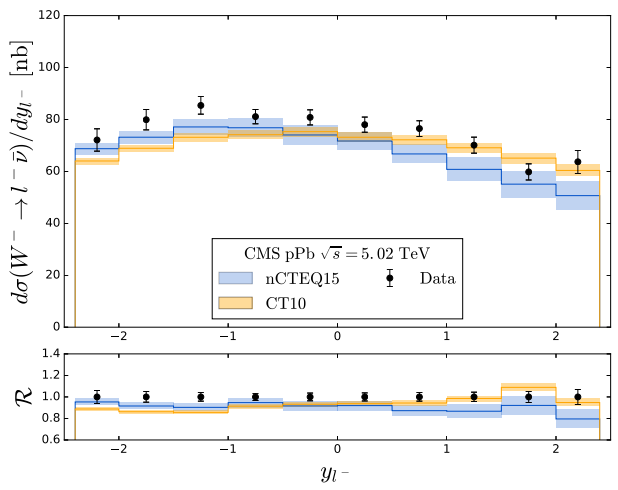

(b) $\mathrm{CMS} W^{-}$

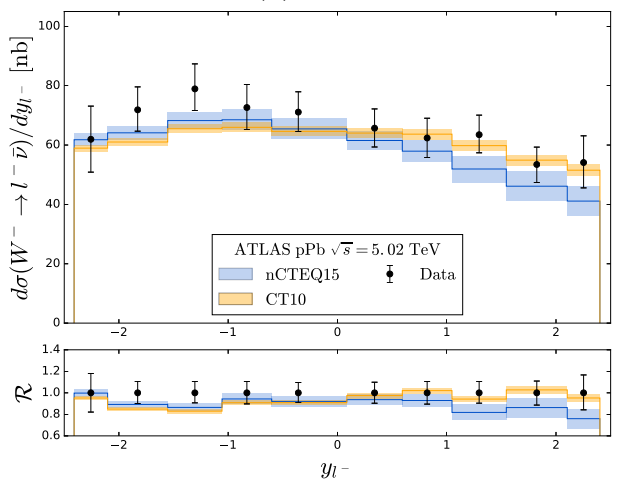

(d) ATLAS $W^{-}$

Fig. 3. $W^{ \pm}$production in $p \mathrm{~Pb}$ collisions at the LHC from CMS (upper plots) and ATLAS (lower plots) compared with predictions from nCTEQ15 nPDFs and CT10 proton PDFs.

In both $W^{+}$and $W^{-}$cases, we see a common pattern. The low rapidity $\left(y_{l^{ \pm}}<0\right)$ data are well-described by the nPDFs, whereas when we go toward larger rapidities $\left(y_{l^{ \pm}}>0\right)$, the deviations between data and nPDF predictions grow. It can be understood in the following way. If we map the rapidity values to the $x$ of lead nucleus that is probed $^{3}$, we find that the negative rapidities correspond to moderate- $x$ values $(\sim 0.1)$ and positive rapidities to the low- $x$ values $\left(\sim 3 \times 10^{-3}\right)$, see Fig. 4. At the same time, we know that the low- $x$ range of nPDFs is unconstrained by the data currently used in the nPDF fits, so these results come from an extrapolation of the larger- $x$ region.

It is interesting to observe that a delayed shadowing (which shifts the shadowing down to smaller- $x$ values) would improve the comparison of the data with the theory in the larger $y_{l^{ \pm}}$region; this type of behavior was ob-

\footnotetext{
3 This strictly holds only at leading order.
} 


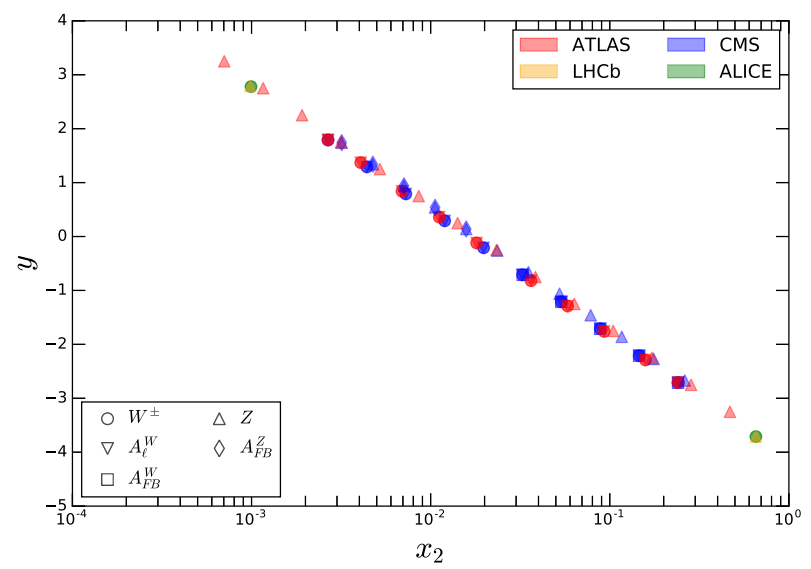

Fig. 4. Kinematic $x$-rapidity plane of lead covered by currently available LHC $p \mathrm{~Pb}$ $W / Z$ production data.

served in the nuclear corrections extracted from the neutrino-DIS charged current data [24-27]. Taking into account the errors from both the experimental data and the theoretical predictions, no definitive conclusions can be drawn at the present time. Nonetheless, this data has the potential to strongly influence the $\mathrm{nPDF}$ fits, especially in the small- $x$ region. This will be even more pronounced with the new data collected at the end of 2016 , where nearly 10 times more statistics were recorded.

\section{Strange contribution}

In order to analyze our results more quantitatively, it is very useful to look at PDF correlations. In particular, we are interested in assessing the importance of the strange quark in our results. We will focus here on the correlations between $W^{+}$and $W^{-}$cross sections, a more comprehensive discussion including $Z$ cross section is presented in [17]. The correlations will be quantified by means of correlation cosine defined in [17, 28]. In our figures, they are plotted as ellipses around central predictions for different PDFs.

Figure 5 shows the correlations of the predicted $W^{+}$and $W^{-}$production cross sections for $p \mathrm{~Pb}$ collisions at the LHC in comparison with the CMS measurements. The same result is displayed in Fig. 6 but split into three different rapidity regions, $y<-1,|y|<1, y>1$. For the proton side, we always use the CT10 PDFs and for the lead side, we examine four cases: (i) nCTEQ15, (ii) CT10, (iii) CT10 PDFs supplemented by the nuclear corrections from EPS09 (CT10+EPS09), and (iv) CTEQ6.1 proton PDFs supplemented by EPS09 nuclear corrections (CTEQ6.1+EPS09). Ad- 
ditionally, to quantify the contribution of the strange quark, we present also results calculated using only 2 quark flavors (one family) $\{u, d\}$. In this way, we eliminate the contribution from the strange PDF (the $c$ and $b \mathrm{PDF}$ contributions are small).

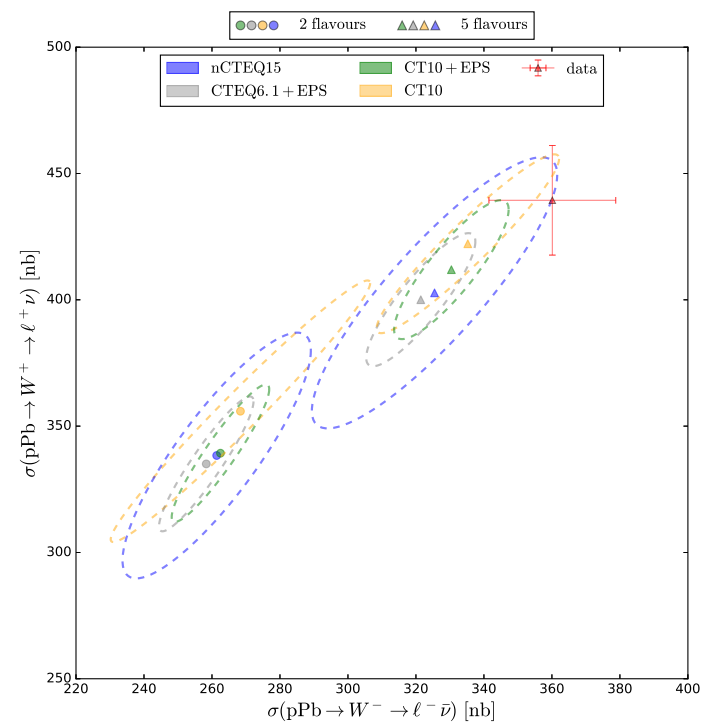

Fig. 5. Comparison of correlations between $W^{+}$and $W^{-}$cross sections for the case when only one family of quarks $\{u, d\}$ is included and when all families are accounted for.

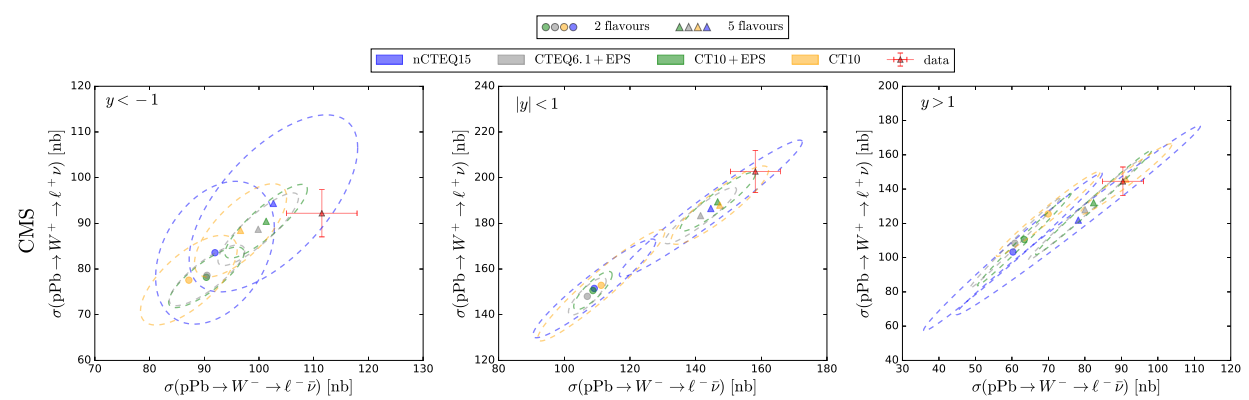

Fig. 6. The same as Fig. 5 but divided into rapidity bins.

The shift of the 2 flavor results compared to the 5 flavor results can be as large as $30 \%$ and reflects the large size of the strange contributions. The strange contributions to $W / Z$ boson production at the LHC are substantial [29] and are primarily responsible for the observed differences among the nuclear results (nCTEQ15, EPS09+CT10, EPS09+CTEQ6.1). On the 
other hand, the observed differences between the 2 flavor proton CT10 and the nuclear (nCTEQ15, EPS09) results accurately represent the nuclear corrections associated with these quantities. Indeed, the nCTEQ15 and EPS09+CTEQ6.1 results are generally very close due to the fact that the CTEQ6.1 and nCTEQ15 baseline PDFs are very similar.

As we review these correlation plots, there are a number of general features which we can identify. As we move from negative $y$ to positive $y$, we move from high $x$ where the nPDFs are well-constrained to small $x$ where the nPDFs have large uncertainties (still underestimated). Thus, it is encouraging that at $y<-1$, we uniformly find the nuclear predictions yield larger cross sections than the proton results (without nuclear corrections) and thus lie closer to the LHC data. Conversely, for $y>1$, we find the nuclear predictions yield smaller cross sections than the proton results. This situation suggests a number of possibilities.

First, the large nPDF uncertainties in the small- $x$ region could be improved using the LHC data.

Second, the lower nPDF cross sections are partly due to the nuclear shadowing in the small- $x$ region; if, for example, this shadowing region were shifted to even lower- $x$ values, this would increase the nuclear results. Such a shift was observed in Refs. [24-26] using charged current neutrino-DIS data, and this would move the nuclear predictions at $y>1$ toward the LHC data.

Finally, we note that measurements of the strange quark asymmetry [30] indicate that $s(x) \neq \bar{s}(x)$ which is unlike what is used in the current nPDFs; this would influence the $W^{ \pm}$cross sections separately as (at leading-order) $W^{+} \sim \bar{s} c$ and $W^{-} \sim s \bar{c}$. As the strange PDF has a large impact on the $W^{ \pm} / Z$ measurements, this observation could provide incisive information on the individual $s$ and $\bar{s}$ distributions.

\section{Impact of the data on nPDFs}

Ultimately, to see the impact of the LHC vector boson data on the nCTEQ15 PDFs, we will perform a new global analysis including these data. This work is ongoing but in the meantime, we try to estimate these effects by employing the reweighting method [31-34].

In this exercise, we use the Giele-Keller (GK) weight supplemented by the tolerance criterion $T$ used in the nCTEQ15 fit

$$
w_{k}=\frac{e^{-\frac{1}{2} \chi_{k}^{2} / T}}{\frac{1}{N_{\text {rep }}} \sum_{i}^{N_{\text {rep }}} e^{-\frac{1}{2} \chi_{k}^{2} / T}}, \quad \chi_{k}^{2}=\sum_{j}^{N_{\text {data }}} \frac{\left(D_{j}-T_{j}^{k}\right)^{2}}{\sigma_{j}^{2}},
$$

where $\chi_{k}^{2}$ represents the $\chi^{2}$ of the data sets considered in the reweighting procedure for a given replica $k$. This definition of the weight has been 
shown to reproduce the full Hessian fit [33, 34]; as such, it is an appropriate choice for PDFs produced using the Hessian framework. More details of the reweighting procedure can be found in our detailed study [17]. Here, we only note that after the reweighting, the PDF-dependent observables and their errors can be computed as weighted sums

$$
\begin{aligned}
\langle\mathcal{O}\rangle_{\text {new }} & =\frac{1}{N_{\text {rep }}} \sum_{k=1}^{N_{\text {rep }}} w_{k} \mathcal{O}\left(f_{k}\right), \\
\delta\langle\mathcal{O}\rangle_{\text {new }} & =\sqrt{\frac{1}{N_{\text {rep }}} \sum_{k=1}^{N_{\text {rep }}} w_{k}\left(\mathcal{O}\left(f_{k}\right)-\langle\mathcal{O}\rangle\right)^{2}} .
\end{aligned}
$$

As an example, we consider the reweighting using the CMS $W^{ \pm}$production data from $p \mathrm{~Pb}$ collisions [9] (these data have the smallest uncertainties among the currently available vector boson $p \mathrm{~Pb}$ data). In this example, we use rapidity distributions of charged leptons originating from the decay of both $W$ bosons and we employ $N_{\text {rep }}=10^{4}$ replicas.

In Fig. 7, we show the comparison of the data to theory before and after the reweighting procedure ${ }^{4}$. As expected, we see that after the reweighting procedure, the description of the data is improved. This is true for both the $W^{+}$(left panel) and $W^{-}$(right panel) cases. We can quantify the improvement of the fit by examining the $\chi^{2} / N_{\text {data }}$ for the individual distributions. For the $W^{+}$case, the $\chi^{2} / N_{\text {data }}$ is improved from 5.07 before reweighting to 3.23 after reweighting. Similarly, for $W^{-}$, the $\chi^{2} / N_{\text {data }}$ is improved from 4.57 to 3.44 . The amount of change due to the reweighting procedure should be proportional to the experimental uncertainties of the incorporated data. For $W^{ \pm}$production investigated here, the uncertainties are quite substantial, and the effects are compounded by the lack of correlated errors.

Still, even with the current data uncertainties, we can see that the improvement of the data description after the reweighting procedure is limited and the resulting $\chi^{2} / N_{\text {data }}$ values are not satisfactory. This is caused by considerably underestimated nPDF error bands, especially in the positive rapidity region. As mentioned before, the low- $x$ (large rapidity) region of the current $\mathrm{nPDFs}$ is extrapolated as there are no constrains form data prior to the LHC measurements. The underestimation of the errors is a result of too restrictive parametrization form used in the $\mathrm{nPDF}$ analyses which, however, is necessary to obtain stable fits.

\footnotetext{
${ }^{4}$ We note here the difference of PDF uncertainties compared to the plots presented in the previous sections. This is caused by the use of the $68 \%$ C.L. errors compared to the standard nCTEQ15 90\% C.L. errors which were used earlier. This holds for all reweighting plots.
} 

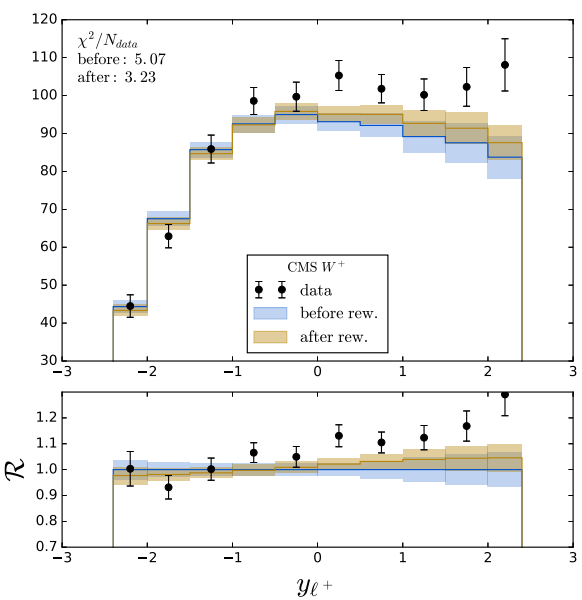

(a) $W^{+}$
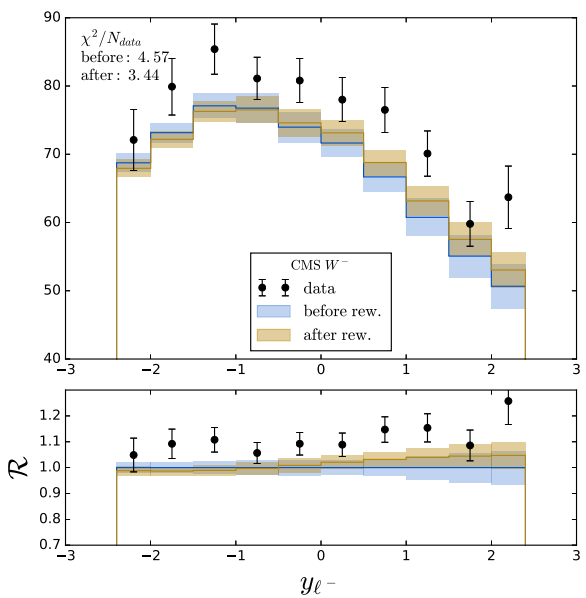

(b) $W^{+}$

Fig. 7. Comparison of data and theory before and after the reweighting using CMS $W^{ \pm}$data for the nCTEQ15 PDFs.

This exercise shows that it is mandatory to release some of the assumptions and use more flexible parameterizations to fully accommodate the vector boson LHC data in nPDF global fits. This is also confirmed by the new EPPS16 analysis [20].

\subsection{Strange contribution}

We have shown that strange distribution is important for the $W / Z$ production at the LHC. This clearly suggests that one of the reasons we have difficulties accommodating $W^{ \pm}$data in the current reweighting exercise is the lack of proper estimates of strange distribution errors. Due to the lack of data, the strange PDF is not fitted in the nCTEQ15 and other nuclear analyses, but it is fixed to be proportional to the light sea distribution $\bar{u}+\bar{d}$. We try to address this problem doing a dedicated fit (referred to as strALL2c) where nCTEQ15 analysis is extended by including neutrino di-muon data $[35]^{5}$. These data can put limited constraints on the strange PDF allowing us to free some of the corresponding fit parameters, and consequently provide more realistic error bars. This can be seen in Fig. 8 where predictions for the CMS $W^{ \pm}$data for this new fit with extra strange flexibility is compared to the original prediction for the nCTEQ15 PDFs. We can

${ }^{5}$ The neutrino di-muon data are often used in proton PDF analyses to constrain strange distribution. They are, however, rarely used in the nuclear PDF fits because of the unanswered question about the compatibility of the charge lepton and neutrino nuclear corrections. 
see a substantial increase of the error bars in the positive rapidity (low $x$ ) region, this is the extrapolation region, where nuclear PDF errors are underestimated to a large extent.

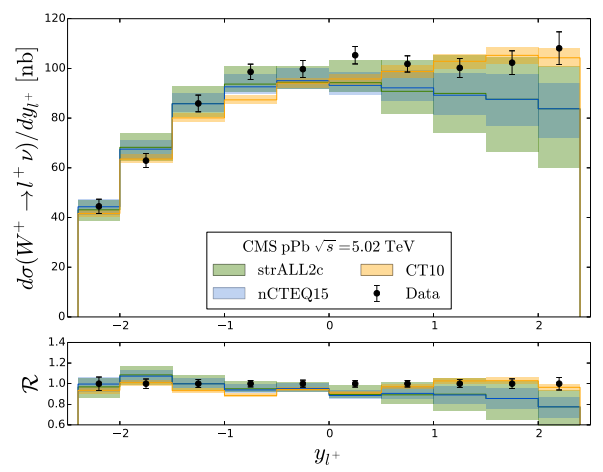

(a) $W^{+}$

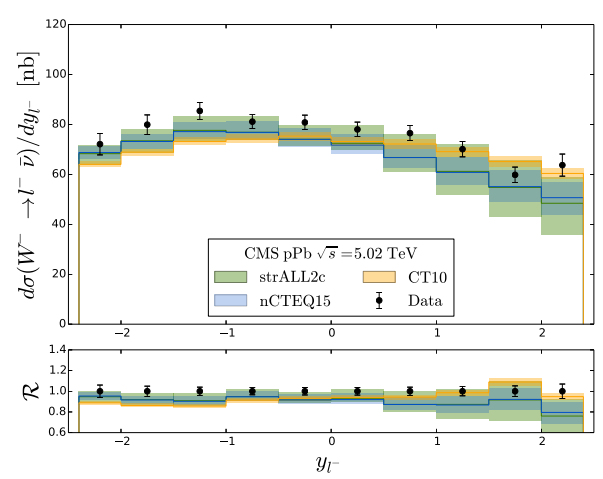

(b) $W^{+}$

Fig. 8. $W^{ \pm}$production in $p \mathrm{~Pb}$ collisions at the LHC from CMS compared with predictions from nCTEQ15, CT10, and strALL2c (nCTEQ15 with additional strange flexibility) PDFs.

To finish this exercise, we perform the reweighting on the new strALL2c PDFs and see if the additional flexibility allows for more reliable reweighting. In Fig. 9, we present the comparison of the data to theory before and after the reweighting procedure using the strALL2c fit. We can see that, indeed, the extra flexibility in strange distribution (higher errors) allowed for more

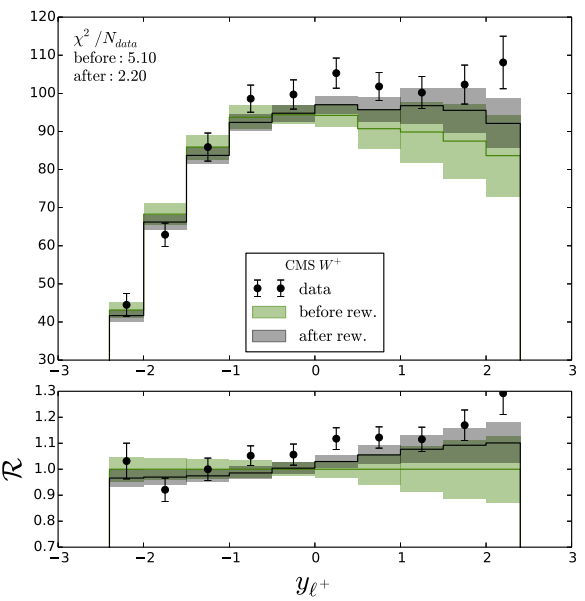

(a) $W^{+}$

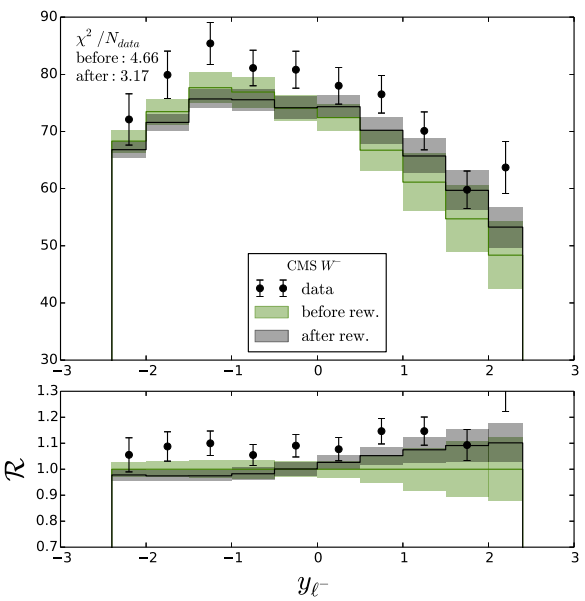

(b) $W^{+}$

Fig. 9. Comparison of data and theory before and after the reweighting using CMS $W^{ \pm}$data for the strALL2c PDFs (nCTEQ15 with additional strange flexibility). 
effective reweighting. The $\chi^{2} / N_{\text {data }}$ for $W^{+}$case is now 2.20 and for $W^{-}$, it is 3.17. Especially in the case of the $W^{+}$boson, the improvement compared to nCTEQ15 reweighting is over 1 point per data point.

This result shows that we are going in the right direction, however, the obtained $\chi^{2} / N_{\text {data }}$ are still relatively large and new fit with even more flexibility is needed to properly incorporate the LHC vector boson data.

\section{Conclusions}

We have presented a study of vector boson production in lead collisions at the LHC. These data are of particular interest for nPDF determinations. A comparison with the LHC proton data provides a direct probe of nuclear corrections for large $A$ values in a kinematic $\left\{x, Q^{2}\right\}$ range very different from the nuclear corrections extracted from fixed-target measurements.

Our study has demonstrated the importance of the strange distribution for the vector boson production at the LHC, possibly even pointing to a nuclear strangeness asymmetry $(s(x)>\bar{s}(x))$. More importantly, it showed that the currently used nuclear strange distributions are not adequate and the characteristic underestimation of errors can cause problems with the description of the LHC data.

This sensitivity to the strange distribution and heavier flavors can provide important information on the nuclear flavor decomposition, which is invaluable for a precise nPDF determination.

Intriguingly, the large rapidity $W / Z$ data seem to prefer nuclear PDFs with no shadowing or delayed shadowing at small $x$, similar to what has been observed in neutrino DIS.

\section{REFERENCES}

[1] R.D. Ball et al., Nucl. Phys. B 867, 244 (2013)

[arXiv:1207.1303 [hep-ph]].

[2] K. Kovarik et al., Phys. Rev. D 93, 085037 (2016) [arXiv:1509.00792 [hep-ph]].

[3] K.J. Eskola, H. Paukkunen, C.A. Salgado, J. High Energy Phys. 0904, 065 (2009) [arXiv:0902.4154 [hep-ph]].

[4] M. Hirai, S. Kumano, T.-H. Nagai, Phys. Rev. C 76, 065207 (2007) [arXiv:0709.3038 [hep-ph]].

[5] H. Paukkunen, C.A. Salgado, J. High Energy Phys. 1103, 071 (2011) [arXiv:1010.5392 [hep-ph]].

[6] G. Aad et al. [ATLAS Collaboration], Phys. Rev. C 92, 044915 (2015) [arXiv:1507.06232 [hep-ex]]. 
[7] V. Khachatryan et al. [CMS Collaboration] Phys. Lett. B 759, 36 (2016) [arXiv: 1512.06461 [hep-ex]].

[8] R. Aaij et al. [LHCb Collaboration], J. High Energy Phys. 1409, 030 (2014) [arXiv: 1406.2885 [hep-ex]].

[9] V. Khachatryan et al. [CMS Collaboration], Phys. Lett. B 750, 565 (2015) [arXiv: 1503.05825 [hep-ex]].

[10] ATLAS Collaboration, ATLAS-CONF-2015-056.

[11] K.J. Senosi [ALICE Collaboration], PoS Bormio2015, 042 (2015) [arXiv:1511.06398 [hep-ex]].

[12] G. Aad et al. [ATLAS Collaboration], Phys. Rev. Lett. 110, 022301 (2013) [arXiv: 1210.6486 [hep-ex]].

[13] S. Chatrchyan et al. [CMS Collaboration], J. High Energy Phys. 1503, 022 (2015) [arXiv:1410.4825 [nucl-ex]].

[14] G. Aad et al. [ATLAS Collaboration], Eur. Phys. J. C 75, 23 (2015) [arXiv: 1408.4674 [hep-ex]].

[15] S. Chatrchyan et al. [CMS Collaboration], Phys. Lett. B 715, 66 (2012) [arXiv:1205.6334 [nucl-ex]].

[16] N. Armesto et al., Eur. Phys. J. C 76, 218 (2016) [arXiv:1512.01528 [hep-ph]].

[17] A. Kusina et al., arXiv:1610.02925 [nucl-th].

[18] D. de Florian, R. Sassot, P. Zurita, M. Stratmann, Phys. Rev. D 85, 074028 (2012) [arXiv:1112.6324 [hep-ph]].

[19] P. Ru, S.A. Kulagin, R. Petti, B.-W. Zhang, Phys. Rev. D 94, 113013 (2016) [arXiv: 1608.06835 [nucl-th]].

[20] K.J. Eskola, P. Paakkinen, H. Paukkunen, C.A. Salgado, Eur. Phys. J. C 77, 163 (2017) [arXiv:1612.05741 [hep-ph]].

[21] F. Arleo, E. Chapon, H. Paukkunen, Eur. Phys. J. C 76, 214 (2016) [arXiv: 1509.03993 [hep-ph]].

[22] H.-L. Lai et al., Phys. Rev. D 82, 074024 (2010) [arXiv: 1007.2241 [hep-ph]].

[23] S. Quackenbush, R. Gavin, Y. Li, F. Petriello, Comput. Phys. Commun. 184, 209 (2013) [arXiv:1201.5896 [hep-ph]].

[24] K. Kovarik et al., Phys. Rev. Lett. 106, 122301 (2011) [arXiv: 1012.0286 [hep-ph]].

[25] I. Schienbein et al., Phys. Rev. D 80, 094004 (2009) [arXiv:0907.2357 [hep-ph]].

[26] S.X. Nakamura et al., Rep. Prog. Phys. 80, 056301 (2017) [arXiv: 1610.01464 [nucl-th]].

[27] N. Kalantarians, JPS Conf. Proc. 12, 010028 (2016).

[28] P.M. Nadolsky et al., Phys. Rev. D 78, 013004 (2008) [arXiv:0802.0007 [hep-ph]]. 
[29] A. Kusina et al., Phys. Rev. D 85, 094028 (2012) [arXiv:1203.1290 [hep-ph]].

[30] D. Mason et al. [NuTeV Collaboration], Phys. Rev. Lett. 99, 192001 (2007).

[31] W.T. Giele, S. Keller, Phys. Rev. D 58, 094023 (1998) [arXiv:hep-ph/9803393].

[32] R.D. Ball et al. [NNPDF Collaboration], Nucl. Phys. B 849, 112 (2011) [Erratum ibid. 855, 926 (2012)] [arXiv:1012.0836 [hep-ph]].

[33] H. Paukkunen, P. Zurita, J. High Energy Phys. 1412, 100 (2014) [arXiv:1402.6623 [hep-ph]].

[34] N. Sato, J.F. Owens, H. Prosper, Phys. Rev. D 89, 114020 (2014) [arXiv:1310.1089 [hep-ph]].

[35] M. Goncharov et al. [NuTeV Collaboration], Phys. Rev. D 64, 112006 (2001) [arXiv:hep-ex/0102049]. 